\title{
Escala de Apoio Social (MOS-SSS): Proposta de Normatização com Referência nos Itens
}

\author{
Daniela Sacramento Zanini ${ }^{1, *}$ \\ Orcid.org/0000-0003-2515-2820 \\ Evandro Morais Peixoto ${ }^{2}$ \\ Orcid.org/0000-0003-1007-3433 \\ Tatiana de Cássia Nakano ${ }^{3}$ \\ Orcid.org/0000-0002-5720-8940
}

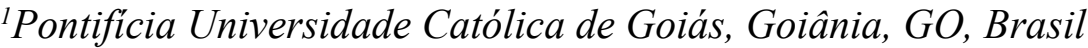 \\ ${ }^{2}$ Universidade de Pernambuco, Garanhuns, PE, Brasil \\ ${ }^{3}$ Pontifícia Universidade Católica de Campinas, Campinas, SP, Brasil
}

\section{Resumo}

A Escala de Apoio Social (MOS-SSS) visa avaliar em que medida a pessoa conta com o apoio de outras para enfrentar situações estressantes. É uma escala amplamente utilizada, contudo há uma escassez de estudos que avaliem suas normas interpretativas. O presente estudo visa estabelecer normas e pontos de corte para MOS-SSS. Participaram deste estudo 998 pessoas de ambos os sexos, com idades entre $12 \mathrm{e}$ 73 anos $(M=27,18, D P=9,90)$, de diferentes estratos amostrais $(61,7 \%$ estudantes universitários; $14,7 \%$ pacientes em tratamento para infertilidade; $10,1 \%$ pacientes em tratamento para transtorno alimentar $\mathrm{e}$ obesidade; $8,7 \%$ submetidos à cirurgia bariátrica; e 4,8\% pacientes em tratamento para queimaduras). O modelo de créditos parciais foi empregado para estabelecimento de normas com referência nos itens, por meio do procedimento Mapa de item-pessoas. Foram estabelecidas as normas com base no procedimento de transformação da pontuação total em theta, o que possibilitará a aplicação prática dos resultados obtidos. Os dados obtidos servirão como como norma de referência para as pontuações obtidas em diferentes estudos para profissionais que utilizam esta escala.

Palavras-chave: Interação social, saúde, normas, teoria de resposta ao item, teste psicológico.

\section{The Social Support Scale (MOS-SSS): Standardizing with Item References}

\begin{abstract}
The Social Support Scale (MOS-SSS) aims to assess the extent to which the person has the support of others to face stressful situations. It is a widely used scale, but there is a shortage of studies evaluating its interpretative rules. This study aims to establish standards and cutoffs for MOS-SSS. The study included 998 people of both sexes, aged between 12 and 73 years $(M=27.18, S D=9.90)$, from different sampling strata $(61.7 \%$ college students, $14.7 \%$ of patients in treatment infertility; $10.1 \%$ of patients in
\end{abstract}

Endereço para correspondência: Rua 9, n. 769, Apto. 702, Setor Oeste, Goiânia, GO, Brasil 623215-1371. Fone: (62)99841664.E-mail: dazanini@yahoo.com, epeixoto_6@hotmail.com e tatiananakano@hotmail.com 
treatment for eating disorder and obesity, $8.7 \%$ of patients after bariatric surgery, and $4.8 \%$ of patients treated for burns). The model of partial credits was used to establish standards relating to items by the procedure item-persons map. Rules have been established based on the processing procedure of the total score theta, which will enable the practical application of the results. The data will serve as a reference standard for the scores obtained in different studies for professionals who use this scale.

Keywords: Social interaction, health, standards, item response theory, psychological test.

\section{Escala de Apoyo Social (MOS-SSS): Propuesta de Normalización con Referencia a los Items}

\section{Resumen}

La Escala de Apoyo Social (MOS-SSS) tiene como objetivo evaluar el grado en que la persona tiene el apoyo de los demás para hacer frente a diferentes situaciones estresantes. Es una escala de larga utilización, pero hay una escasez de estudios para evaluar sus normas interpretativas. Este estudio tiene por objeto establecer las normas y los puntos de corte para la MOS-SSS. El estudio incluyó 998 personas de ambos sexos, con edades comprendidas entre 12 y 73 años $(M=27.18, D E=9.90)$, de diferentes estratos de muestreo $(61,7 \%$ estudiantes universitarios, $14,7 \%$ pacientes en tratamiento infertilidad; $10,1 \%$ pacientes en tratamiento por trastornos de la alimentación y obesidad, $8,7 \%$ pacientes pós cirugía bariátrica, y 4,8\% pacientes tratados por quemaduras). Se utilizó el modelo de créditos parciales para establecer normas relativas a los ítems por el procedimiento del mapa ítem-persona. Las normas se han establecido sobre la base del procedimiento de tramitación de la theta total de puntos, lo que permitirá la aplicación práctica de los resultados. Los datos servirán como como patrón de referencia para las puntuaciones obtenidas en diferentes estudios para los profesionales que utilizan esta escala.

Palabras clave: Interación social, normas, teoria de respuesta al ítem, test psicológico.

Desde os anos de 1970 o interesse no estudo do efeito das relações sociais sobre a saúde das pessoas passou a ser alvo de pesquisadores de diversas áreas. Seu estudo ganhou fôlego por meio das pesquisas que demonstravam como as relações sociais, em suas diferentes formas, estavam relacionadas a melhores preditores de saúde nos indivíduos, melhor adaptação social, protegendo contra os efeitos negativos do estresse e diminuindo a morbidade e a mortalidade (Cobb, 1976; Dantas, Araújo, Paulino, \& Maia, 2012; Griep, Chor, Faerstein, Werneck, \& Lopes, 2005; Sherbourne \& Stewart, 1991). Entre os tipos de relações interpessoais que influenciam na saúde, Sherbourne e Stewart (1991) diferenciam os conceitos de rede e apoio social. Rede social é o grupo de pessoas com as quais o indivíduo mantém contato ou alguma forma de vínculo social. Sua avaliação revelaria o nível de integração social do indivíduo.

Apoio social, por outro lado, pode ser considerado como algo que diz respeito aos recur- sos postos a disposição por outras pessoas em situações de necessidade. Sua avaliação, além de indicar o quão integrado socialmente é este individuo, também indica o grau com que relações interpessoais correspondem a determinadas funções e oferecem suporte ao indivíduo em momentos de crise ou readaptação. Em outras palavras, rede social pode ser concebida como a estrutura social pela qual o apoio é fornecido (Griep, 2003), enquanto o apoio social refere-se a dimensão funcional ou qualitativa da rede social (Griep et al., 2005).

Gonçalves, Pawlowski, Bandeira e Piccinini (2011) realizaram um levantamento nos indexadores Indexpsi, Pepsic, SciELO e Lilacs com o objetivo de investigar como o apoio social tem sido avaliado em estudos brasileiros entre os anos de 1987 a 2007. Os autores encontraram 59 estudos que contemplavam a avaliação de apoio social em amostras brasileiras e indicaram um aumento nos últimos anos no número de estudos brasileiros que incluem a avaliação do apoio so- 
cial. Estes estudos utilizam uma diversidade de métodos de avaliação do apoio social, embora com a predominância do uso de técnicas de entrevistas para investigar, em especial, o apoio recebido e o apoio percebido. Ainda assim, quando utilizam de escalas para avaliação do apoio social, a grande maioria usou a Escala de Apoio Social do Estudo Pró- Saúde em detrimento das outras. Por fim, os autores apontam para a escassez de informação sobre fidedignidade e validade dos instrumentos originais e das adaptações realizadas para o Brasil nas pesquisas estudadas e ressaltam a necessidade de estudos sistemáticos que estabeleçam a fidedignidade, validade $\mathrm{e}$ normatização desses instrumentos a fim de que possam ser considerados adequados para uso no Brasil.

\section{A Escala de Apoio Social MOS-SSS (Social Support Scale)}

Desenvolvida para o Medical Outcome Stu$d y$ (Sherbourne \& Stewart, 1991), a Escala de Apoio Social MOS-SSS (Social Support Scale) visa avaliar em que medida a pessoa conta com o apoio de outras para enfrentar diferentes situações em sua vida. Embora desenvolvida para aplicação em pacientes crônicos, seu uso se estendeu a diferentes populações devido a sua fácil aplicação (19 itens que são respondidos por meio de uma escala tipo Likert de cinco pontos), e boa qualidade psicométrica demonstrada em diferentes estudos com populações e contextos diversos (Gómez-Campelo et al, 2014; Pais-Ribeiro \& Ponte, 2009). Em estudo de busca por evidências de validade, o instrumento original apresentou uma estrutura interna composta por cinco fatores, identificados como apoio social do tipo emocional, informacional, material, afetivo e de interação social positiva, e índices de consistência interna superiores a 0,91 (Sherbourne $\&$ Stewart, 1991).

Posteriormente esta escala foi traduzida e adaptada para uso no Brasil por Griep et al. (2005). Essa versão foi utilizada em diferentes estudos nacionais conduzidos com o instrumental. Zanini, Verolla-Moura e Queiroz (2009) investigaram a estrutura fatorial do instrumento em uma amostra composta por 129 estudantes universitários e encontraram quatro dimensões (apoio emocional/ informacional, interação social, apoio material e apoio social afetivo), semelhantes ao modelo original, composto por cinco fatores. A diferença situa-se no fato de que, nas análises brasileiras, dois fatores agruparam-se em um só (emocional e informacional). A consistência interna revelou bons índices de adequação da medida para a população estudada, com os índices alfas variando entre 0,76 e 0,95.

Outro estudo desenvolvido por Griep et al. (2005) junto a 4.030 funcionários técnico-administrativos efetivos de uma universidade, apontou a existência de três fatores: afetivo + interação social positiva, emocional + informação e matéria. Novamente verifica-se a presença dos elementos similares, mas agora com o agrupamento dos fatores referentes aos fatores interação social positiva e apoio afetivo. Em relação aos índices de consistência interna estes variavam entre 0,83 e 0,92 .

Por fim, Zanini e Peixoto (2016) avaliaram a pertinência dos diferentes modelos fatoriais propostos para MOS-SSS em estudos brasileiros com diferentes grupos amostrais. Os resultados revelaram melhor qualidade de ajustamento do modelo composto por quatro fatores (apoio emocional/ informacional, interação social, apoio material e apoio social afetivo) os mesmos encontrados em Zanini et al. (2009). Além disso, demostrou-se a invariância total (configural, métrica e escalar) do modelo fatorial quando comparados os diferentes estratos amostrais: estudantes universitários, pacientes em tratamento para infertilidade, pacientes em tratamento para transtorno alimentar e obesidade, pacientes submetidos à cirurgia bariátrica e pacientes em tratamento para queimaduras, assim como quando separados por sexo, o que indica a equivalência do modelo fatorial, cargas fatoriais e medias obtidas nos itens entre os grupos amostrais (controlando-se as diferenças nas variáveis latentes). De acordo com Santos e Primi (2014) a verificação da invariância dos modelos métrico e escalar equivalem, respectivamente, a inexistência de Função diferencial dos itens (DIF) no parâmetro discriminação (a) e no parâmetro dificuldade (b) em Teoria de Resposta ao Item (TRI). 
Por fim, por meio do Modelo de Créditos Parciais (MCP) os autores verificaram que os itens avaliavam a porção central dos construtos latente propostos pelos fatores, com índices de dificuldade próximos do valor médio (ancorado em 0) e índices ajustes adequados (Infit/Outfit), bem como indicadores de precisão, coeficientes alfa de Cronbach, que variavam entre $0,83 \mathrm{e}$ 0,92 . Contudo, ainda que estudos de busca por evidencias de validade e outras propriedades psicométricas já tenham sido desenvolvidos com a versão brasileira do instrumento, e pese sua larga utilização, ainda são escassos os estudos que ofereçam sentido psicológico aos resultados obtidos junto a MOS-SSS. Em geral, a análise dos resultados é realizada por meio da pontuação bruta em estudos correlacionais para análise comparativa intra-amostra (ex.: Yun, Kang, Lim, Oh, \& Son, 2010) ou análise dos níveis de pontuações por meio da distribuição de seu próprio estudo (Andrade et al., 2005).

Andrade e colaboradores (2005) classificaram o apoio social segundo a distribuição da pontuação da amostra para cada um dos fatores do instrumento, classificando o resultado em tercis: baixa, média e alta pontuação para cada fator. Esse tipo de análise, embora seja apropriada estatisticamente e sirva aos objetivos das pesquisas específicas não permitem a comparação entre estudos. Ou seja, permite uma análise intra-estudo eficaz, porém não é eficiente para a análise do nível de apoio social desta amostra em relação aos demais estudos e/ou estratos amostrais. Tal dificuldade seria resolvida a partir da condução de estudos de normatização da escala, a fim de que resultados passíveis de interpretação para o referido instrumento de pesquisa possam ser conhecidos.

Com objetivo de oferecer contribuição para o preenchimento desta lacuna recorreu-se, nesta pesquisa, ao procedimento mapa de item (Item Map) com base na TRI que têm sido apontado como uma alternativa para a realização da normatização referenciada nos itens (McClarty, 2013; Primi, Wechsler, Nakano, Oakland, \& Guzzo, 2014; Wyse, 2013). Segundo Embretson e Reise (2000), dentre os benefícios deste procedimento destaca-se o acesso à maneira como as pessoas se descrevem por meio do conteúdo presente nos itens, de modo a fornecer significado psicológico aos resultados de uma pessoa. Para tanto são estimados, por meio da TRI, os parâmetros dos itens (dificuldade, identificado pela letra $b$ ) e das pessoas (nível de traço latente, identificado pela letra grega $\theta$ ). Por meio da associação destes parâmetros, procedimento denominado calibração, a interpretação passa a ser referenciada no item.

A implementação do processo de normatização referenciada nos itens, baseia-se nos seguintes pressupostos: (a) conhecimento das propriedades dos instrumentos tais como estrutura interna e estrutura da escala Likert, (b) conhecimento dos parâmetros dos itens (índices de dificuldade e ajuste) e das pessoas (nível de traço latente); (c) calibração do parâmetro das pessoas e dos itens (alocação dos parâmetros numa escala comum); e (d) análise do mapa de itens para definição das interpretações referenciadas nos itens. Considerando-se que os passos (a) e (b) com a mesma amostra foram descritos em um estudo anterior (Zanini \& Peixoto, 2016), a presente pesquisa contemplará os resultados referentes aos passos (c) e (d). Assim, o presente estudo teve como objetivos: estabelecer normas interpretativas e pontos de corte para Escala de apoio social (MOS-SSS) por meio da análise do mapa de item (Item Map), com base na Teoria de Resposta ao Item (TRI).

\section{Método}

\section{Participantes}

A amostra para esta pesquisa foi composta por 998 participantes de ambos os sexos $(65,1 \%$ mulheres), com idades entre 12 e 73 anos $(M=$ $27,18, D P=9,90)$, sendo $61,7 \%$ estudantes universitários; $14,7 \%$ pacientes em tratamento para infertilidade; $10,1 \%$ pacientes em tratamento para transtorno alimentar e obesidade; 8,7\% pacientes submetidos à cirurgia bariátrica e $4,8 \%$ pacientes em tratamento para queimaduras. Quanto ao nível de escolaridade dos pacientes, $4,9 \%$ dos participantes tinham ensino fundamental, $61,32 \%$ ensino superior incompleto, $16,5 \%$ ensino médio, $15,03 \%$ ensino superior completo. 
Em relação ao estado civil, 64,4\% eram solteiros, $31,6 \%$ casados, $3,2 \%$ separado ou divorciado e $0,9 \%$ viúvo. Quanto à origem dos participantes, os estudantes universitários eram provenientes de quatro instituições de ensino superior, duas privadas localizadas no interior de São Paulo, uma privada localizada na cidade de São Paulo e uma pública localizada no interior de Goiás. O estrato amostral composto por pacientes era proveniente de ambulatórios localizados no interior de Goiás.

\section{Instrumento}

Escala de Apoio Social (MOS-SSS) desenvolvida para o Medical Outcomes Study (MOS-SSS) por Sherbourne e Stewart (1991) e adaptada para a população brasileira pela equipe de Griep no estudo Pró Saúde no Rio de Janeiro (Griep et al., 2005). O instrumento é composto por 19 questões que o participante deve responder a partir de uma instrução inicial: "Se você precisar, com que frequência conta com alguém?", assinalando uma das cinco respostas possíveis de acordo com uma escala Likert de cinco pontos: 0 ("nunca"); 1 ("raramente"); 2 ("às vezes"); 3 ("quase sempre") e 4 ("sempre"). Embora não existam estudos de normatização para população brasileira assume-se que maiores índices no escore total dos respectivos fatores indicam maior percepção do tipo de apoio. Destaca-se que para a soma do escore total não é necessário a inversão de nenhum item (Zanini et al., 2009).

\section{Procedimentos}

Análise de Dados. Por meio do MCP, método de estimação Joint maximum likelihood, estimou-se os mapas de pessoas-itens, para interpretação com referência no item (Embretson $\&$ Reise, 2000). Vale destacar que os mapas foram desenvolvidos separadamente para cada um dos quatro fatores que compuseram a MOS-SSS, em respeito aos pressupostos de unidimensionalidade e independência local exigidos por esse modelo. As análises foram realizadas no pacote estatístico WINSTEPS versão 3.30.0 (Linacre, 2015) utilizando-se da amostra total. Esta deci- são foi pautada nos pressupostos de invariância já observado por Zanini e Peixoto (2016), bem como no fato do procedimento mapa de itens comparar as características dos sujeitos (nível estimado de theta) com as características dos itens da escala (dificuldade), diferenciando-se assim da comparação dos escores apresentados por um sujeito com aqueles observados por um grupo normativo (Embretson \& Reise, 2000).

Coleta de Dados. A coleta de dados foi realizada de forma individual nos grupos amostrais específicos nas próprias unidades de saúde que atendiam e de forma coletiva nos estudantes universitários na própria sala de aula. Em todos os grupos amostrais o tempo médio de resposta ao questionário foi de 15 minutos. Todos os participantes da pesquisa foram convidados a participar mediante explicação dos objetivos e risco de sua participação na pesquisa. A todos foi lido o Termo de Consentimento Livre e Esclarecido (TCLE) e após sua assinatura iniciou-se a coleta de dados. Foi garantido o sigilo dos dados assim como a possibilidade de retirada da participação em qualquer momento da pesquisa sem qualquer ônus, seguindo-se todos os procedimentos éticos preconizados pela APA e Resolução 196/1996.

\section{Resultados}

A Figura 1 apresenta o mapa dos itens referente ao fator emocional/informacional. A apresentação e interpretação dos mapas de itens segue uma padronização. Desta forma, nos mapas os itens são apresentados em ordem de dificuldade, o item mais facilmente endossado pelos participantes está localizado na base do mapa, seguindo uma ordem crescente de dificuldade, o mais difícil se localiza no topo do mapa. Em relação aos símbolos utilizados, o símbolo “:”, representa o ponto de transição na probabilidade de escolha da alternativa de resposta, em função do nível de theta do sujeito (por exemplo: em que nível de theta o sujeito provavelmente deixa de assinalar a alternativa 1 para assinalar a alternativa 2). Além disso, três linhas são apresentadas abaixo do mapa: a primeira denominada 'pessoas' que representa a frequência de pessoas 
localizadas em cada ponto da escala de $\theta$ (sendo os valores lidos na vertical), a segunda linha 'estatísticas' representa as estatísticas descritivas de $\theta$ dos participantes, sendo a média representada pela letra (M), um desvio padrão (S) e dois desvios padrões (T). E a terceira linha 'percentil' indica o percentual de sujeitos alocados em cada ponto da escala de $\theta$.

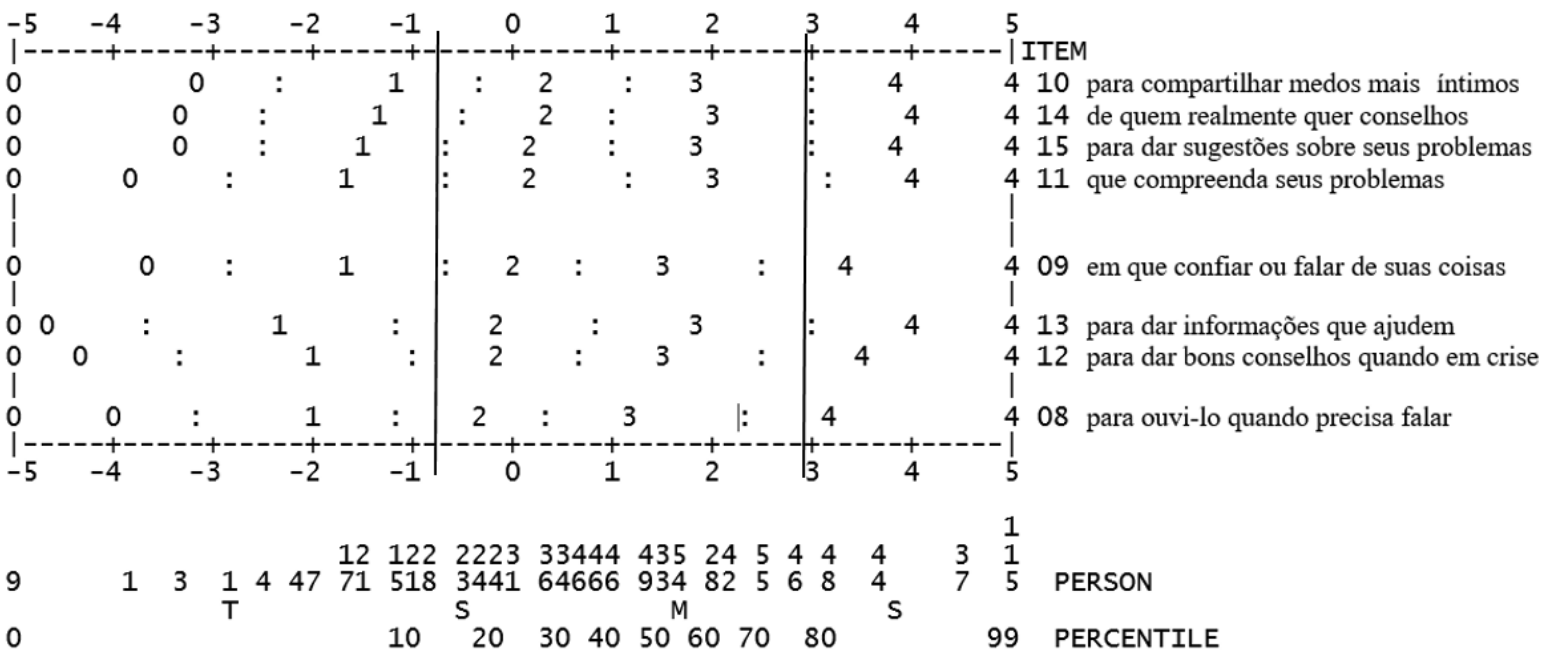

Figura 1. Mapa de itens do fator Emocional/Informacional. Nota. Itens abreviados, transmitindo somente a ideia principal.

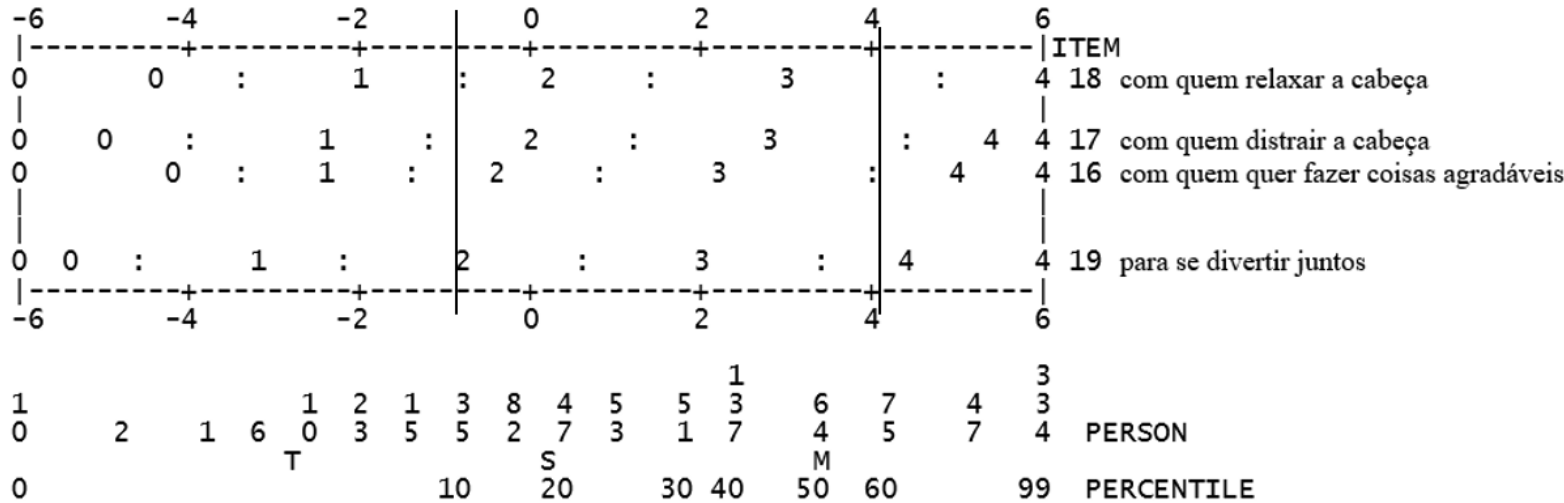

Figura 2. Mapa de itens do fator Interação social. Nota. Itens abreviados, transmitindo somente a ideia principal.

Notadamente, maior número de pessoas apresentou nível de $\theta$ entre $-0,6(\mathrm{~S})$ e 3,8 (S) aproximadamente, indicando que as pessoas tendem a concordar mais do que discordar do conteúdo dos itens que compõem este fator, o que resulta na distribuição deslocada para o lado positivo da escala. Vale destacar que o fator avalia a percepção de apoio Emocional/Informacional recebido de outras pessoas, neste sentido a fragilidade da pessoa avaliada, teoricamente, é indicada por baixos escores nestes itens.
Conforme observado na Figura 1, pessoas com nível de $\theta$ médio, 1,6 aproximadamente, tendem a ter pessoas com quem contar "quase sempre" nas situações descritas pelo conteúdo dos itens, endossando a alternativa 3 na escala Likert para todos os itens que compõem o fator. Pessoas com nível de $\theta$ um desvio padrão abaixo da média, 0,6 aproximadamente, tendem a endossar a alternativa 2 na escala Likert na faixa de item que compreender desde o item 08 até o item 14, informando ter "as vezes" alguém com 
quem contar nas situações expressas pelos itens. No entanto, apresentam $50 \%$ de probabilidade de endossarem as alternativas 1 "raramente" ou 2 "as vezes" quanto a ter alguém de quem realmente quer conselhos (item 14), e endossar a alternativa 1 "raramente" tenho alguém para compartilhar medos mais íntimos (item 10). No outro polo da escala, pessoas com nível de $\theta$ um desvio padrão acima da média, 3,8 aproximadamente, tendem endossar a alternativa 4 da escala Likert "sempre" ter alguém com quem contar nas situações descritas pelos itens.

Quanto ao fator Interação social, observa-se na Figura 2 maior dispersão quando ao nível de $\theta$ das pessoas, desta forma a escala varia de -6 a 6 . Neste fator, pessoas com nível de $\theta$ médio, aproximadamente 3,2, apresentam $50 \%$ de probabilidade de endossarem as alternativas 3 "quase sempre" ou 4 "sempre" quanto a ter alguém para divertir juntos, bem como tendem a endossar a alternativa 3 "quase sempre" quanto a ter alguém com quem quer fazer coisas agradáveis (item16), com quem distrair a cabeça (item 17), e com quem relaxar a cabeça (item 18). Enquanto pessoas com nível de $\theta$ um desvio padrão abaixo da média, 0,2 aproximadamente, tendem a endossar a alternativa 2 , indicando ter "as vezes" alguém com quem contar para as situações descritas pelos itens. Notadamente, trata-se de um fator que contém itens mais fáceis de serem endossados, haja vista que pessoas com níveis de $\theta$ médios já podem endossar a alternativa 4 que representa maior intensidade no traço avaliado.

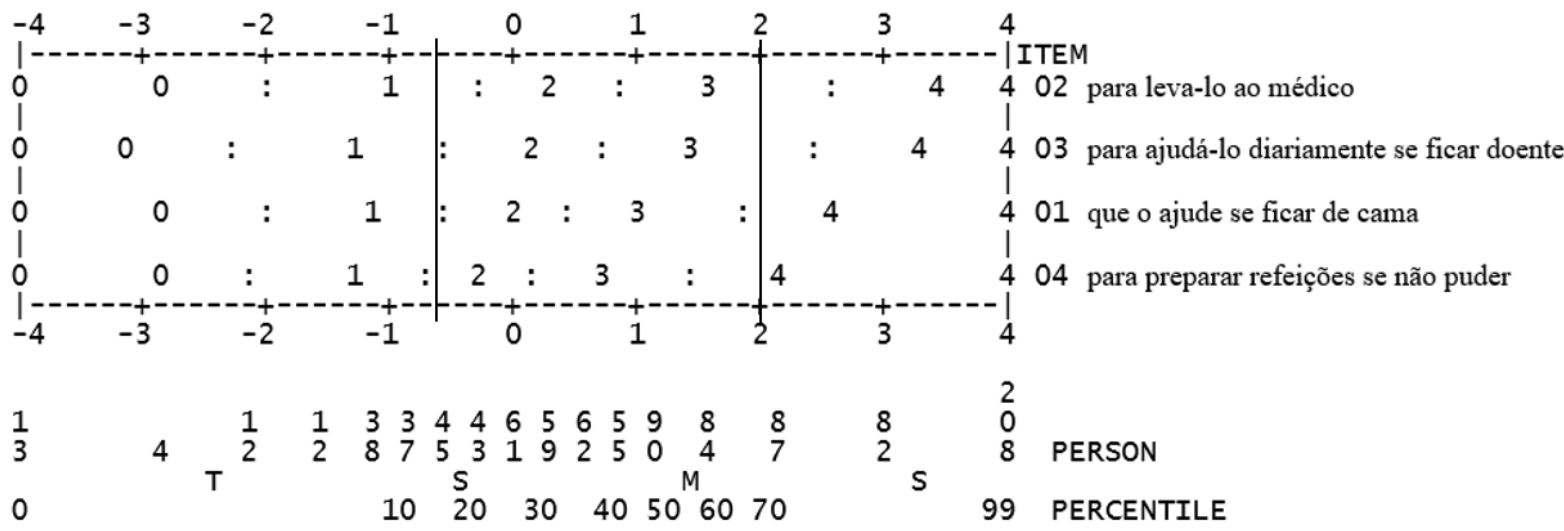

Figura 3. Mapa de itens do fator Material.

Nota. Itens abreviados, transmitindo somente a ideia principal.

No fator Material (Figura 3), maior número de pessoas apresentou nível de $\theta$ entre $-0,8$ e 3 aproximadamente, indicando como nos outros fatores, que as pessoas tendem a concordar mais do que discordar do conteúdo dos itens. Assim, pessoas com nível de $\theta$ próximo da média, aproximadamente 1,5 , tendem a endossar a alternativa 3 da escala Likert "quase sempre" quanto a ter alguém com quem contar diante as situações descritas pelos itens que compõem o fator. Pes- soas com nível de $\theta$ um desvio padrão abaixo da média tendem a endossar a alternativa 2 para a faixa de itens que compreende os itens $04,01 \mathrm{e}$ 03 (em ordem de dificuldade), e endossar a alternativa 1 escala da Likert "raramente" tenho alguém com quem contar para leva-lo ao médico (item 02). Por outro lado, pessoas com níveis de $\theta$ um desvio padrão acima da média tendem a endossar a alternativa 4 "sempre" em todos os itens que compõem o fator. 


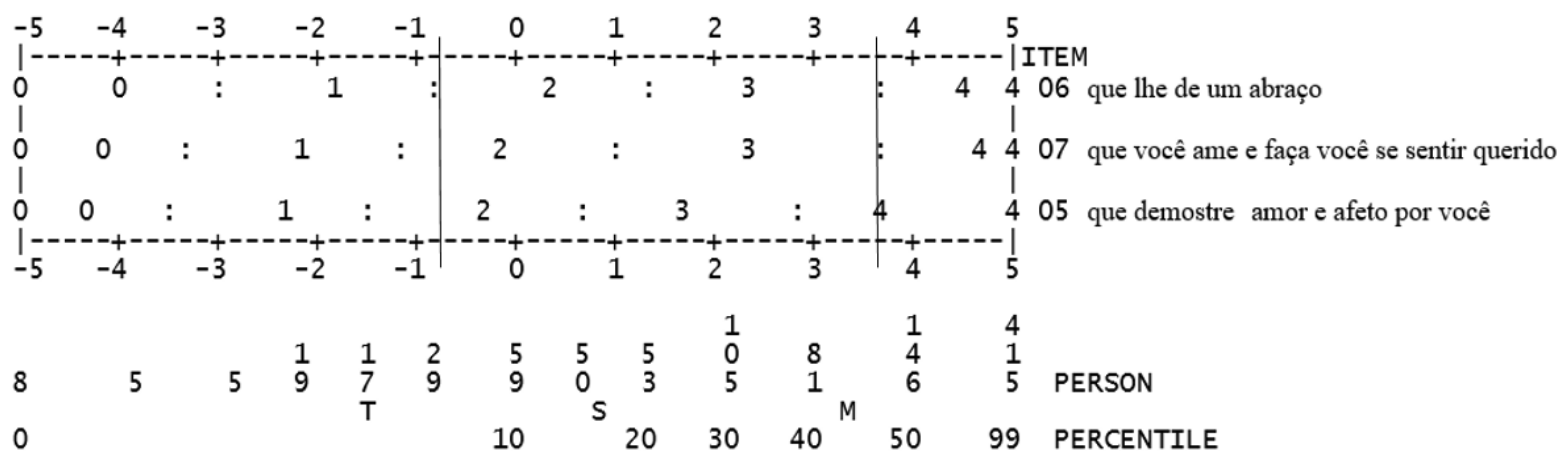

Figura 4. Mapa de itens do fator Afetivo.

Nota. Itens abreviados, transmitindo somente a ideia principal.

Por fim, o fator Afetivo, Figura 4, também se caracteriza por contar com itens que apresentam maior probabilidade de serem endossados. Pessoas com nível de $\theta$ próximos da média 3,3 , tendem a endossar a alternativa 4 "sempre" quanto a contar com alguém que demostre amor e afeto (Item 05) e alternativa 3 da escala Likert (quase sempre) quanto a contar com alguém que ame e o faça sentir querido (item 07) e que lhe de um abraço (item 06). Já uma pessoa com nível de $\theta$ um desvio padrão abaixo da média tende a endossar a alternativa 3 "quase sempre" para o item 5, e a alternativa 2 "as vezes" para os itens 07 e 06 (em ordem de dificuldade).

A fim de possibilitar a comparação dos resultados de uma pessoa avaliada com o padrão de autodescrição, por meio do conteúdo dos itens, observado nos diferentes pontos da escala de theta empregou-se o procedimento que objetiva a transformação de escore total em $\theta$. Os indicadores desse procedimento são apresentados na Tabela 1, são eles: os possíveis escores obtidos por meio dos quatro fatores que compõem a MOS-SSS e a equivalência em nível de $\theta$ destes escores.

A partir do procedimento de transformação de escore total em nível de $\theta$ torna-se possível os estabelecimentos dos pontos de corte com base no escore total e, portanto, a aplicação prática dos conhecimentos oriundos do procedimento mapa de itens-pessoas (Primi et al., 2014). Conforme Tabela 1, observa-se que para o fator emocional/informacional os escores podem variar entre 0 e 32 com níveis de $\theta$ que variavam entre $-5,97$ e 5,74 . Com base no conteúdo dos itens estabeleceu-se que pessoas com nível de $\theta$ até $-0,7$ (ou escore total 12) devem ser classificadas como baixo nível de percepção de apoio social do tipo Emocional/informacional, uma vez que apresentaram maior probabilidade de endossarem a alternativa 2 "as vezes" para a faixa de item que compreende os itens 08,12 e 13 , e a alternativa 1 "raramente" para os itens que compreende os itens $09,11,15,14$ e 10. Pessoas com nível de $\theta$ entre $-0,6$ e 2,9 (ou escore total entre 13 e 28 ) devem ser classificados como nível de apoio médio, uma vez que apresentam maior probabilidade de endossarem as alternativas 2 ou 3 nos itens que compõem o fator. Por fim, pessoas com níveis de $\theta$ superiores a 3 (ou escore total superior 13) devem ser classificados como nível de apoio social alto, haja vista a probabilidade de endossarem a alternativa 4 "sempre" para todos os itens.

Para o fator Interação social os escores variaram entre 0 e 16 , e níveis de $\theta$ associados que variavam entre -6,18 e 6,72. Estabeleceu-se que pessoas com nível de $\theta$ até $-0,9$ (ou escore total 6) devem ser classificadas com nível de percepção de apoio social do tipo Interação social baixo, visto probabilidade de endossarem a alternativa 2 "as vezes" para o item 4 , e a alternativa 1 "raramente" para os itens 01,03 e 02. Pessoas com nível de $\theta$ entre $-0,8$ e 3,99 (ou escore total entre 7 e 13) devem ser classificadas com nível de percepção de apoio médio devido a maior probabilidade de endossarem as alternativas 2 "as vezes" e 3 quase sempre" nos quatro itens que compõem o fator. Já as pessoas com nível de $\theta$ superiores a 4 (ou escore total igual 
Tabela 1

Transformação do Escore Total em Theta

\begin{tabular}{|c|c|c|c|c|}
\hline \multirow{2}{*}{ Escore total } & \multicolumn{4}{|c|}{ Theta } \\
\hline & Emocional/Inform. & Interação social & Material & Afetivo \\
\hline 0 & $-5,97$ & $-6,18$ & $-4,16$ & $-5,19$ \\
\hline 1 & $-4,68$ & $-4,8$ & $-2,89$ & $-3,83$ \\
\hline 2 & $-3,86$ & $-3,83$ & $-2,1$ & $-2,87$ \\
\hline 3 & $-3,33$ & $-3,14$ & $-1,59$ & $-2,15$ \\
\hline 4 & $-2,91$ & $-2,53$ & $-1,2$ & $-1,48$ \\
\hline 5 & $-2,56$ & $-1,97$ & $-0,87$ & $-0,79$ \\
\hline 6 & $-2,25$ & $-1,4$ & $-0,57$ & $-0,08$ \\
\hline 7 & $-1,96$ & $-0,82$ & $-0,29$ & 0,65 \\
\hline 8 & $-1,7$ & $-0,22$ & $-0,02$ & 1,37 \\
\hline 9 & $-1,45$ & 0,39 & 0,25 & 2,11 \\
\hline 10 & $-1,21$ & 1,03 & 0,54 & 2,93 \\
\hline 11 & $-0,98$ & 1,72 & 0,84 & 4 \\
\hline 12 & $-0,76$ & 2,49 & 1,18 & 5,44 \\
\hline 13 & $-0,55$ & 3,32 & 1,59 & \\
\hline 14 & $-0,34$ & 4,2 & 2,12 & \\
\hline 15 & $-0,14$ & 5,29 & 2,95 & \\
\hline 16 & 0,07 & 6,72 & 4,25 & \\
\hline 17 & 0,27 & & & \\
\hline 18 & 0,47 & & & \\
\hline 19 & 0,67 & & & \\
\hline 20 & 0,87 & & & \\
\hline 21 & 1,07 & & & \\
\hline 22 & 1,28 & & & \\
\hline 23 & 1,5 & & & \\
\hline 24 & 1,72 & & & \\
\hline 25 & 1,96 & & & \\
\hline 26 & 2,22 & & & \\
\hline 27 & 2,5 & & & \\
\hline 28 & 2,82 & & & \\
\hline 29 & 3,2 & & & \\
\hline 30 & 3,7 & & & \\
\hline 31 & 4,48 & & & \\
\hline 32 & 5,74 & & & \\
\hline
\end{tabular}

Nota. As diferentes tonalidades de cores correspondem aos pontos de corte estabelecidos para os fatores baixo, médio e alto. 
ou superior a 14) devem ser classificadas com nível de apoio alto, visto a maior probabilidade de endossarem a alternativa 4 "sempre" para os itens 04 e 01 , e alternativa 3 "quase sempre" para os itens 03 e 02 .

Para o fator Material os escores variaram entre 0 e 16, com nível $\theta$ associados que variavam entre $-4,16$ a 4,25. Estabeleceu-se que pessoas com nível de $\theta$ até $-0,7$ (ou escore total até 6) devem ser classificadas com nível de percepção de apoio do tipo Material, uma vez que apresentam maior probabilidade de endossarem a alternativa 2 "as vezes" para o item que apresenta menor intensidade do traço 04 , e a alternativa 1 para os outros itens: 01, 03 e 02 . Pessoas com níveis de $\theta$ entre -0,6 e 1,99 (ou escore total entre 7 e 13) devem ser classificados com nível de percepção de apoio médio devido maior probabilidade de endossarem as alternativas 2 "as vezes" e 3 "quase sempre" nos quatro itens que compõem o fator. Por fim, pessoas com nível de $\theta$ igual ou superior a 2 (ou escores total igual ou superior a 14) devem ser classificados com nível de percepção de apoio alto, notadamente tendem a endossar a alternativa 3 "quase sempre" nos itens com menor intensidade do construto "04-para preparar refeições se não puder" e "01- que ajude se ficar de cama", e alternativa 3 "quase sempre" para os itens mais intensos "03- para ajudá-lo diariamente se ficar doente" e para leva-lo ao médico".

Para o fator Afetivo os escores variaram entre 0 e 12, com nível $\theta$ associados que variavam entre $-5,19$ a 5,44. Estabeleceu-se que pessoas com nível de $\theta$ até $-0,8$ (ou escore total 4) devem ser classificadas com nível de percepção de apoio do tipo afetivo baixo, uma vez que apresentam maior probabilidade de endossarem a alternativa dois nos itens menos intensos "05- que demostre amor e afeto por você" e "07- que você ame e faça você se sentir querido", e a alternativa 1 "raramente" para o item "06- que de um abraço". Pessoas com nível de $\theta$ entre $-0,7$ e 3,7 (ou escore total entre 05 e 10) com nível de percepção de apoio médio, devido a maior probabilidade de endossarem as alternativas 2 "as vezes" e 3 "quase sempre" os três itens que compões o fator. Por fim pessoas com níveis de $\theta$ igual ou superior 3,8 (ou escore total igual ou superior 11) devem ser classificadas com nível de percepção de apoio alto, pois notadamente tendem a endossar a alternativa 4 "sempre" em todos os itens.

\section{Discussão}

O presente estudo buscou estabelecer normas interpretativas e pontos de corte para MOS-SSS diante a população brasileira. Para tanto empregou ao MCP, mais especificamente o procedimento Mapa de itens-pessoas para interpretação referenciada no item. Os resultados indicam níveis de dificuldades diferentes para cada um dos fatores analisados pelo instrumento. Os fatores com itens com maiores níveis de dificuldade e, portanto, em que os participantes tendem a emitir menor número de respostas afirmativas são o apoio social do tipo Emocional e Informacional e o Apoio Material. Contudo, os fatores apoio social do tipo Interação Social positiva e Afetivo apresentaram-se mais fáceis e portanto, com maior probabilidade do participante responder de forma positiva. Estes dados indicam que para a amostra estudada há uma maior percepção da existência de pessoas em sua rede social que lhes amem (Apoio afetivo) e com quem podem ter momentos de prazer e relaxamento (Iteração Social), contudo, percebem menos a possibilidade de terem com quem contar para dividir suas preocupações e sentimentos (Apoio Emocional), solicitar informações (Informacional) e apoiar-lhes com medidas práticas (Apoio Material).

Cabe ressaltar que esta escala avalia a percepção da existência de apoio social, mas não sua efetividade nem quantidade. Desta forma, os dados demonstram que os participantes percebem mais o apoio afetivo contudo, não apontam a quantidade de pessoas disponíveis para este apoio. Pode ser que a percepção avaliada por este instrumento esteja relacionada a uma única relação social, por exemplo entre mãe e filha. E que esta relação seja percebida como suficientemente potente para que o participante indique alto nível de existência deste tipo de apoio. Esta discussão já foi apontada em outros estudos sobre o tema que medem percepção de apoio e não o apoio propriamente dito (Schwarzer \& Knoll, 2007). Em contrapartida, a percepção do tipo 
de apoio social tal como o Material pode estar influenciado pelo nível de demanda dos participantes. Desta forma, apesar de sua existência, pode não ser avaliado como suficiente a ponto de produzir uma resposta positiva no instrumento. Tais aspectos devem ser levados em consideração na hora da interpretação dos dados e estão em consonância com o que a literatura sobre o tema tem descrito (Queiroz \& Zanini, 2011).

Além disso, a análise das diferenças nos níveis de theta e a probabilidade de endosso dos itens que compõem cada um dos fatores corroboram esta discussão. Por exemplo, ao analisar mais detalhadamente a Figura 1 com a distribuição dos níveis de dificuldade de cada item, verifica-se importante diferença conceitual entre o item 08 (em que medida conta com alguém para ouvi-lo caso precise) considerado o mais fácil e, portanto, com maior probabilidade de respostas muito positivas, e os itens 10 (alguém para compartilhar seus medos mais íntimos) e 14 (alguém de quem realmente quer conselhos). Nestes itens (10 e 14) a probabilidade de respostas muito positivas diminui demonstrando que embora os participantes tenham avaliado mais fácil a existência de apoio social do tipo Emocional/Informacional não são todos os tipos de apoio que são facilmente percebidos. Embora tenha-se maior disponibilidade de pessoas para dar conselhos, não são de todas que se quer este conselho e nem sempre isso significa que uma disponibilidade de pessoas para compartilhar medos íntimos. Essa discussão volta a evidenciar a distinção entre rede social e rede de apoio social (Zanini et al., 2009) e a discussão sobre os níveis e tipos de interação social e seu impacto no bem-estar das pessoas (Kraut et al., 1998).

Por fim, o presente estudo possibilita a compreenssão qualitativa da diferença entre pessoas localizadas em diferentes pontos da escala de theta. A título de exemplo pode-se verificar a maneira como pessoas com níveis de theta iguais a $-2,0$ e 2 se descrevem por meio do conteúdo dos itens dispostos nos quatro fatores que compõem o instrumento. Por meio da Figura 1 verifica-se que pessoas com níveis de tetha igual a -2 tendem a "raramente" ter uma pessoa que lhe ofereça apoio emocional/informacional, o que significa raramente ter alguém para ouvi-los quando precisam falar (item 08), para dar bons conselhos quando estão em crise (item 12), para dar informações que ajudem (13), em quem confirmar ou falar de suas coisas (item 09), que compreenda seus problemas (item 11), para dar sugestões sobre seus problemas (item 15), de quem realmente quer conselhos (item 14), ou mesmo para partilhar medos mais íntimos (item 10). Pessoas com níveis de theta iguais a 0 tendem, "às vezes", a contar com o apoio de alguém nestas situações. Por fim, pessoas com níveis de theta igual a 2 se descrevem como alguém que "quase sempre" tem alguém com quem contar em situações como essas.

No fator Interação social (Figura 2) a mesma dinâmica pode ser observada. Neste fator pessoas com níveis de theta igual a -2 indicavam "às vezes" ter alguém para se divertir juntos (item 19), e raramente alguém com quem fazer coisas agradáveis (itens 16), com quem distrair a cabeça (item 17) ou com quem relaxar a cabeça (item 18). Pessoas com níveis de theta igual a 0 tendem a raramente ter com quem contar nestas situações apresentadas pelos itens, enquanto pessoas com níveis de theta igual a 2 tendem a ter "quase sempre" com quem contar para a vivência de tais situações.

No fator Material (Figura 3), pessoas com níveis de theta igual -2 indicam que "raramente" tem com quem contar para preparar refeições se não puderem (item 4) ou para ajudá-los diariamente se ficarem doentes (item 3), ao passo que ficaram em dúvida em endossar as alternativas "nunca" ou "raramente" em casos de ter alguém com quem contar que os ajudem se ficarem de cama (item 1) ou para levá-los ao médico (item 2). Frente estas mesmas situações, pessoas com níveis de theta igual a 0 tendem a indicar ter "às vezes" com quem contar. Pessoas com níveis iguais a 2 tendem a sempre ter com quem contar para preparar refeições se não puderem (item 4) ou para ajudá-los diariamente se ficarem doentes (item 3), ao passo que "quase sempre" teriam com quem contar que os ajudem se ficarem de cama (item 1) ou para levá-los ao médico (item 2).

Por fim, no fator Afetivo (Figura 4), pessoas com níveis de theta igual a -2 indicam raramen- 
te ter à disposição alguém que lhes demonstre amor e afeto (item 5), que lhes faça sentir querido (item 7) ou que lhes dê um abraço (item 6). Pessoas com níveis de theta igual a 0 indicam ter "às vezes" alguém que lhes ofereça os tipos de apoio afetivo descritos nos itens. Já as pessoas com níveis de theta igual a 2 indicam ter "quase sempre" alguém que lhes ofereça estes tipos de apoio.

Em suma, o presente estudo ofereceu os parâmetros para interpretação das pontuações na escala MOS-SSS com referência no conteúdo dos itens. Esta interpretação pode ser realizada especialmente tomando a Tabela 1 como localizador da pontuação do respondente e seu correspondente na escala de theta. Contudo, os dados deste estudo devem ser tomados com precaução pois, embora apresentem análise estatísticas atuais e que visam minimizar os efeitos de erros amostrais, a amostra utilizada não foi selecionada de forma probabilística e, portanto, não representa a população brasileira. Apesar disso, o presente estudo pode ser de grande valia para psicólogos e profissionais da área que queiram fazer uso deste instrumento e obter parâmetros acerca de como as pessoas, localizadas em diferentes pontos na escala de theta, se descrevem a partir do conteúdo dos itens que compõem este instrumento.

\section{Referências}

Andrade, C. R., Chor, D., Faerstein, E., Griep, R. H., Lopes, C., \& Fonseca, M. J. M. (2005). Apoio social e auto-exame das mamas no Estudo Pró-Saúde. Cadernos de Saúde Pública, 21(2), 379-386. doi.org/10.1590/S0102$-311 \times 2005000200004$

Cobb, S. (1976). Social support as a moderator of life stress. Psychosomatic. Medicine, 38(5), 300314. Retrieved from https://campus.fsu.edu/ bbcswebdav/institution/academic/social_sciences/sociology/Reading\%20Lists/Mental\%20 Health\%20Readings/Cobb-PsychosomaticMed-1976.pdf

Dantas, M. M. C., Araújo, P. C. B., Paulino, B. S., \& Maia, E. M. C. (2012). Avaliação do apoio social e de sintomas depressivos em mães de bebês prematuros hospitalizados. Psicologia em
Revista, 18(1), 90-106. doi.org/10.5752/P.16789563.2012v18n1p90

Embretson, S. E., \& Reise, S. P. (2000). Item response theory for psychologists. Mahwah, NJ: Lawrence Erlbaum.

Gómez-Campelo, P., Pérez-Moreno, E., Burgos-Lunar, C., Bragado-Álvarez, C., Jiménez-García, R., \& Salinero-Fort, M. (2014). Psychometric properties of the eight-item modified Medical Outcomes Study Social Support Survey based on Spanish outpatients. Quality of Life Research, 23(7), 2073-2078. doi:10.1007/s11136014-0651-6

Gonçalves, T. R., Pawlowski, J., Bandeira, D. R., \& Piccinini, C. A. (2011). Avaliação de apoio social em estudos brasileiros: Aspectos conceituais e instrumentos. Ciência \& Saúde Coletiva, 16(3), 1755-1769. doi:https://dx.doi. org/10.1590/S1413-81232011000300012

Griep, R. H. (2003). Confiabilidade e validade de instrumentos de medida de rede social e de apoio social utilizados no Estudo Pró-Saúde (Tese de Doutorado não publicada, Escola Nacional de Saúde Pública, Fundação Oswaldo Cruz, Rio de Janeiro, RJ, Brasil).

Griep, R. H., Chor, D., Faerstein, E., Werneck, G. L., \& Lopes, C. S. (2005). Validade de constructo de escala de apoio social do Medical Outcomes Study adaptada para o português do Estudo Pró-Saúde [Construct validity of the Medical Outcomes Study's social support scale adapted to Portuguese in the Pró-Saúde Study]. Cadernos de Saúde Pública, 21(3), 703-714. doi:10.1590/ S0102-311X2005000300004

Kraut, R., Patterson, M., Lundmark, V., Kiesler, S., Mukophadhyay, T., \& Scherlis, W. (1998). Internet paradox: A social technology that reduces social involvement and psychological well-being?. American Psychologist, 53(9), 1017-1031. doi:http://dx.doi.org/10.1037/0003066X.53.9.1017

Linacre, J. M. (2015). A user's guide to Winsteps Ministep: Rasch-model computer programs. Retrieved from http://www.winsteps.com

McClarty, K. L. (2013). Construct Maps: A tool to organize validity evidence. Measurement, 11, 185-188. doi:10.1080/15366367.2013.857213

Pais-Ribeiro, J. L., \& Ponte, A. C. S. L. C. (2009). Propriedades métricas da versão portuguesa da escala de suporte social do MOS (MOS Social 
Support Survey) com idosos. Psicologia, Saúde \& Doença, 10(2), 163-174. Recuperado em https://sigarra.up.pt/fdup/pt/pub_geral.show_ file?pi_gdoc_id=557613

Primi, R., Wechsler, S. M., Nakano, T. C., Oakland, T., \& Guzzo, R. S. L. (2014). Using Item Response Theory methods with the Brazilian temperament scale for students. Journal of Psychoeducational Assessment, 32(7). doi:https://doi. org/10.1177/0734282914528613

Queiroz, I. P. A. R., \& Zanini, D. S. (2011). Apoio social, coping e suas repercussões no humor depressivo. Estudos: Vida e Saúde (Goiânia), 38(1-3), 133-149. Recuperado em http://seer. pucgoias.edu.br/index.php/estudos/article/ view/1919/1204

Santos, D., \& Primi, R. (2014). Social and emotional development and school learning: A measurement proposal in support of public policy (Technical report for Organization for Economic Cooperation and Development [OCDE] Rio de Janeiro State Education Department [SEEDUC] and Ayrton Senna Institute). São Paulo, SP: Ayrton Senna Institute.

Schwarzer, R., \& Knoll, N. (2007). Functional roles of social support within the stress and coping process: A theoretical and empirical overview. International Journal of Psychology, 42(4), 243 252. doi:10.1080/00207590701396641

Sherbourne, C. D., \& Stewart, A. L. (1991). The MOS Social Support Survey. Social Science Medicine, 32(6), 705-714. Retrieved from http:// www.ncbi.nlm.nih.gov/pubmed/2035047
Wyse, A. E. (2013). Construct Maps as a Foundation for Standard Setting. Measurement, 11, 139170. doi:10.1080/15366367.2013.850287

Yun, E. H., Kang, Y. H., Lim, M. K., Oh, J. K., \& Son, J. M. (2010). The role of social support and social networks in smoking behavior among middle and older aged people in rural areas of South Korea: A cross-sectional study. BMC Public Health, 10, 78. doi:http://doi.org/10.1186/14712458-10-78.

Zanini, D. S., \& Peixoto, E. M. (2016). Social Support Scale (MOS-SSS): Analysis of the Psychometric Properties via Item Response Theory. Paidéia (Ribeirão Preto), 26(65), 359-368. doi:https:// dx.doi.org/10.1590/1982-43272665201612

Zanini, D. S., Verolla-Moura, A., \& Queiroz, I. P. A. R. (2009). Apoio social: Aspectos da validade de constructo em estudantes universitários. Psicologia em Estudo, 14(1), 195-202. doi:10.1590/ S1413-73722009000100023

(C) $\mathrm{O}(\mathrm{s})$ autor(es), 2018. Acesso aberto. Este artigo está distribuído nos termos da Licença Internacional Creative Commons Atribuição 4.0 (http://creativecommons.org/licenses/by/4.0/), que permite o uso, distribuição e reprodução sem restrições em qualquer meio, desde que você dê crédito apropriado ao(s) autor(es) original(ais) e à fonte, fornecer um link para a licença Creative Commons e indicar se as alterações foram feitas.

Recebido: 22/04/2016

$1^{a}$ revisão: 08/09/2016

$2^{a}$ revisão: 03/02/2017

Aceite final: 13/03/2017 